\title{
Approximate Dynamic Programming with Probabilistic Temporal Logic Constraints
}

\author{
Lening $\mathrm{Li}$ and Jie Fu
}

\begin{abstract}
In this paper, we develop approximate dynamic programming methods for stochastic systems modeled as Markov Decision Processes, given both soft performance criteria and hard constraints in a class of probabilistic temporal logic called Probabilistic Computation Tree Logic (PCTL). Our approach consists of two steps: First, we show how to transform a class of PCTL formulas into chance constraints that can be enforced during planning in stochastic systems. Second, by integrating randomized optimization and entropy-regulated dynamic programming, we devise a novel trajectory samplingbased approximate value iteration method to iteratively solve for an upper bound on the value function while ensuring the constraints that PCTL specifications are satisfied. Particularly, we show that by the on-policy sampling of the trajectories, a tight bound can be achieved between the upper bound given by the approximation and the true value function. The correctness and efficiency of the method are demonstrated using robotic motion planning examples.
\end{abstract}

\section{INTRODUCTION}

For safety-critical systems, one main control objective is to ensure desirable system performance with provable correctness guarantees given high-level system specifications. In this work, we study the following problem: Given a stochastic system modeled as a Markov Decision Process (MDP), how to efficiently synthesize a policy that is optimal with respect to a performance criterion while satisfying safety- and missioncritical constraints in temporal logic? For stochastic systems with difficult-to-model dynamics, is it possible to learn such a policy efficiently from sampled trajectories using a black box physics simulator of the system?

MDP planning with temporal logic constraints has been extensively studied. One class of research develops policies that maximize the probability of satisfying given temporal logic specifications [1], [2], [3], [4], [5], [6], [7]. Another class of work considers multiple objectives, including soft constraints-maximizing the total reward-and hard constraints—satisfying safety properties [8], [9]. Among these work, Wang et al.[3] devised the first Approximate Dynamic Programming (ADP) method to solve the problem of maximizing the probability of satisfying temporal logic constraints. The principle of ADP is to introduce policy function approximation, value function approximation, or both (actor-critic methods) [10] such that the number of decision variables originally grows linearly in the state space and action space of the MDP but becomes independent of the size of the MDP. Thus, ADP methods [11] allow one to address the problem of scalability and the lack of a dynamic model in planning for stochastic systems.

L. Li and J. Fu are with the Robotics Engineering Program, Department of Electrical and Computer Engineering, Worcester Polytechnic Institute, Worcester, MA, 01609, USA, lli4, jfu2@wpi.edu
In this work, we develop approximate value iterationa class of ADP methods-in MDP given both soft performance criteria and hard temporal logic constraints. The hard constraints are given by Probabilistic Computation Tree Logic (PCTL) [12], which is used to reason about properties in stochastic systems, for example, "the probability is greater than 0.85 that the goal can be reached with a cost less than 100". Our approach includes two steps: In the first step, we show that a large subclass of PCTL can be equivalently represented by chance constraints over the path distribution in a stochastic system, with appropriately defined cost functions. We introduce the mixing time for Markov chain to approximately verify properties in time-unbounded PCTL using trajectories with finite lengths. It is noted that PCTL involves global properties in a system, which is often hard to enforce using locally optimal policy search methods. In the second step, we develop a chance-constrained approximate value iteration method to solve the planning problem with reward maximization and PCTL constraint satisfaction. In literature, chance-constrained approximate policy iteration method has been developed [13]. We consider approximate value iteration methods to obtain guarantees for global properties in PCTL. This is achieved via integrating randomized optimization and approximate linear programming formulation proposed in [14]. Our samplingbased approximate value iteration method has the following desirable properties: 1) It achieves a tight error bound by weighing the approximation errors over the state space using the state visitation frequencies of an approximately optimal policy; 2) It is probabilistic complete, in the sense that it converges to an approximately optimal policy that satisfies the PCTL constraints with probability one.

The rest of the paper is structured as follows. Section II provides some preliminaries. Section III contains the main results of the paper, including the translation from a subclass of PCTL to chance constraints in MDP and the ADP algorithm. Presented in section IV] are experimental studies with robot motion planning to validate the optimality and correctness of the proposed method. Section $\mathrm{V}$ draws a conclusion.

\section{PReliminaries}

Notation: A finite set $X,|X|$ is the size of $X$, and $\Delta(X)$ denotes the probability simplex in $\mathbb{R}^{|X|}$. Given a distribution $\mu \in \Delta(X), \operatorname{Supp}(\mu)=\{x \in X \mid \mu(x) \neq 0\}$ is the support of $\mu . \mathbb{R}, \mathbb{R}_{+}, \mathbb{R}_{\geq 0}$, and $\mathbb{N}$ are the set of reals, positive reals, nonnegative reals, and natural numbers, respectively. $\mathcal{A P}$ is the set of the atomic propositions. 
A. Markov decision processes and the linear programming formulation of $A D P$

We consider stochastic systems modeled by Markov Decision Processes. An MDP is a tuple $M=\langle S, A, P, r, \mu, \gamma\rangle$, where $S$ is a finite set of states; $A$ is a finite set of actions; $P(\cdot \mid s, a) \in \Delta(S)$ is the probability distribution of the next state by taking action $a$ at state $s ; r: S \times A \rightarrow \mathbb{R}$ is the reward function: $r(s, a)$ represents the immediate reward gained at state $s$ taking action $a ; \mu \in \Delta(S)$ is the initial state distribution; $\gamma \in(0,1]$ is a discount factor. For $s \in S$, we denote $A(s)=\left\{a \in A \mid \exists s^{\prime} \in S, P\left(s^{\prime} \mid s, a\right)>0\right\}$ the set of admissible actions at state $s$.

Given a time step $t \in \mathbb{N}$, a history $h_{t}=s_{0}, a_{0}, s_{1}$, $a_{1}, \ldots s_{t}$ is a finite sequence of state-action pairs prior to time $t$. Let $H_{t}$ be the set of all possible histories prior to time $t$. A decision rule $d_{t}: H_{t} \rightarrow \Delta(A)$ maps a history $h \in H_{t}$ to a distribution $d_{t}(h)$ of admissible actions. Let $\pi=\left(d_{1}, d_{2}, \ldots, d_{N}\right)$ denote a randomized historydependent policy. If $d_{i}=d_{j}=d$ for all $i \neq j$ in a policy, then $\pi=(d, d, \ldots, d)$ is a Markovian, randomized policy. We denote $\Pi$ the set of Markov and randomized policies. A policy $\pi$ induces, from an MDP $M$, a Markov chain $M^{\pi}=X_{0}, A_{0}, X_{1}, A_{1}, X_{2}, \ldots$, where $X_{i}, A_{i}$ are the random variables describing the $i$-th state and action in the chain. We also omit the actions and refer to the $\pi$-induced Markov chain by $M^{\pi}=\left\{X_{n}, n \geq 0\right\}$. It holds that $X_{0} \sim \mu$, $P\left(A_{t}=a_{t} \mid X_{t}=s_{t}\right)=\pi\left(a_{t} \mid s_{0} \ldots s_{t}\right)$, and $P\left(X_{t+1}=\right.$ $\left.s_{t+1} \mid X_{t}=s_{t}\right)=\sum_{a \in A} P\left(s_{t+1} \mid s_{t}, a_{t}\right) \pi\left(a_{t} \mid s_{0} \ldots s_{t}\right)$. We say a state $s \in S$ is a sink state if $P(s \mid s, a)=1$ for all $a \in A(s)$.

A path $\rho=s_{0} s_{1} s_{2} \ldots$ is a finite/infinite sequence of states. Given a policy $\pi$ and path $\rho$, we denote $P^{\pi}(\rho)$ the probability of the path $\rho$ in the $\pi$-induced Markov chain. We denote $\operatorname{Path}^{\pi}(s)$ the set of possible paths starting from state $s$ and following policy $\pi$.

Next, we present the Linear Programming (LP) formulation for optimal planning in MDPs [14]. In planning to maximize an infinite-horizon discounted reward, we have a value function of a policy defined by

$$
V^{\pi}(\mu)=\mathbf{E}\left[\sum_{t=0}^{\infty} \gamma^{t} r\left(X_{t}, A_{t}\right) \mid X_{0} \sim \mu\right] .
$$

The optimal policy $\pi^{*}$ achieves $\pi^{*}=\arg \max _{\pi \in \Pi} V^{\pi}(\mu)$. For maximizing an infinite-horizon discounted reward, there exists an optimal, memoryless policy [15]. The LP [16] solving $\pi^{*}$ is described as follows: Let $V=[V(s)]_{s \in S}$ be a vector of variables, one for each state.

$$
\begin{gathered}
\min _{V} c^{\top} V, \\
\text { subject to: }\left[r(s, a)+\gamma \sum_{s^{\prime}} P\left(s^{\prime} \mid s, a\right) V\left(s^{\prime}\right)\right] \leq V(s), \\
\forall s \in S, a \in A,
\end{gathered}
$$

where $c=\left[c_{1}, \ldots, c_{|S|}\right]^{\top}$ is a vector of nonnegative staterelevance weights, i.e., $c_{i} \geq 0$, for all $i=1, \ldots,|S|$. Once
$V$ is obtained, the optimal policy can be generated using the Bellman equation.

For large-scale MDPs, function approximation of $V$ is introduced to find approximate-optimal policy [14]. Consider a linear function approximator

$$
V(s ; \theta) \approx \sum_{k=1}^{\mathcal{K}} \phi_{k}(s) \theta_{k}=\Phi \theta
$$

where $\phi_{k}(s): S \rightarrow \mathbb{R}, k=1, \ldots, \mathcal{K}$ are preselected basis functions and $\theta=\left[\theta_{1}, \ldots, \theta_{\mathcal{K}}\right]^{\top} \in \mathbb{R}^{\mathcal{K}}$ is a weight vector. The approximate LP is to substitute $V$ with its function approximator $\Phi \theta$ in (1). Let $\theta^{*}$ be the solution. An approximate-optimal policy is computed by $\pi\left(s ; \theta^{*}\right):=$ $\arg \max _{a}\left[r(s, a)+\gamma \sum_{s^{\prime}} P\left(s^{\prime} \mid s, a\right) V\left(s^{\prime} ; \theta^{*}\right)\right]$.

\section{B. Specification: Probabilistic Computation Tree Logic (PCTL) with the reachability reward/cost properties}

PCTL provides syntax and semantics to quantify probabilistic properties [17].

The syntax of PCTL with reachability reward/cost properties [12] is defined as follows:

$$
\begin{aligned}
& \phi:=\text { True }|\alpha| \phi \wedge \phi|\neg \phi| P_{\bowtie p}[\psi] \mid C_{\bowtie m}\left(\diamond^{\leq k} \phi\right) ; \\
& \psi:=\mathrm{X} \phi|\phi \mathrm{U} \leq k \phi| \phi \mathrm{U} \phi,
\end{aligned}
$$

where $\phi$ is a state formula and $\psi$ is a path formula, $\alpha \in \mathcal{A P}$ is an atomic proposition, $k \in \mathbb{N}$ is a nonnegative integer, $\bowtie \in\{\leq, \geq,>,<\}, p \in[0,1]$ is a probability, and $m \in \mathbb{R}$. A path formula $\psi$ is interpreted on paths. $\mathrm{X}$ is the next operator, $\mathrm{U}$ is the until operator, $\mathrm{U} \leq k$ is the bounded until operator, and $\diamond \phi \equiv \operatorname{TrueU} \phi$ is "eventually". $\mathrm{X} \phi$ asserts that the next state satisfies a state formula $\phi . \phi_{1} \mathrm{U} \leq k \phi_{2}$ asserts that $\phi_{2}$ is satisfied within $k$ steps and all preceding states satisfy $\phi_{1}$. $\phi_{1} \cup \phi_{2}$ asserts that $\phi_{2}$ is satisfied some time in the future and all preceding states satisfy $\phi_{1} . \nabla^{\leq k} \phi$ means $\phi$ becomes true in no more than $k$ steps. $P_{\bowtie p}[\psi]$ means that the probability of generating a trajectory that satisfies formula $\psi$ is $\bowtie p$. The reachability reward formula [12] $C_{\bowtie m}\left[\nabla^{\leq k} \phi\right]$ means that the total accumulated rewards/costs along a path of length no greater than $k$ that reaches a state that satisfies $\phi$ are $\bowtie m$, for a predefined reward/cost function. Note that we only consider time-bounded total cost.

Formally, given a $\pi$-induced Markov chain $M^{\pi}$ and a PCTL formula $\phi$, the policy satisfying the constraint $\phi$ given the initial state $s$ is denoted $s \models_{\pi} \phi$.

For ease of understanding, we give several examples of PCTL formulas

- $P_{\geq 0.95}($ TrueU $\leq 10$ reach goal): From any state, the goal can be reached in less than 10 steps with probability at least 0.95 .

- $P_{>0.9}\left(\mathrm{X} C_{\leq 100}\left[\nabla^{\leq 5} \alpha\right]\right)$ : “The probability is larger than 0.9 , that from the next step onward, the system reaches a state satisfying $\alpha$ with a total accumulated cost no greater than 100 in no more than 5 steps.

Our goal is to develop an approximate dynamic programming algorithm that solves the following planning problem: 
Problem 1. Given an MDP $M=\langle S, A, P, r, d, \mu, \gamma\rangle$ and a PCTL formula $\phi$, the goal is to find a policy $\pi$ that solves

$$
\max _{\pi} V^{\pi}(\mu), \text { subject to } M^{\pi} \models \phi .
$$

\section{MAIN RESULT}

Our approach to tackling Problem 1 includes two steps: First, we show that a class of PCTL constraints can be translated into chance constraints. Second, we introduce an approximate value iteration algorithm that solves Problem 1 .

\section{A. Translating PCTL formulas into chance constraints}

Chance constraints are introduced to capture risk-sensitive optimization criteria in MDPs. Let $d: S \times A \rightarrow \mathbb{R}$ be a cost function. For a policy $\pi$, we define the cost of a state $s$ (resp. state-action pair $(s, a)$ ) as the sum of finite-horizon (discounted) total costs encountered by the decision-maker when it starts at state $s$ (resp. state-action pair $(s, a)$ ) and then follows policy $\pi$, as follows:

$$
\begin{gathered}
D(s, a, T ; \pi)=\sum_{t=0}^{T-1} \gamma^{t} d\left(X_{t}, A_{t}\right) \mid X_{0}=s, A_{0}=a ; \\
D(s, T ; \pi)=\sum_{a \in A(s)} D(s, a, T ; \pi) \pi(a \mid s),
\end{gathered}
$$

where $\gamma$ is a discount factor and $T \in \mathbb{N} \cup\{\infty\}$ is a stopping time.

Chance-constrained planning in MDP aims to ensure that for a given initial state $X_{0}$, a confidence level $\beta \in(0,1)$, and cost tolerance $\alpha$, the policy $\pi$ that satisfies

$$
\operatorname{Pr}\left(D\left(X_{0}, T ; \pi\right) \geq \alpha\right) \leq \beta .
$$

Let $\rho=s_{0} s_{1} \ldots \in \operatorname{Path}^{\pi}(s)$, we define $D(\rho ; \pi)=$ $\sum_{t=0}^{|\rho|-1} \gamma^{t} d\left(s_{t}, a_{t}\right)$. Then the chance constraint is equivalently expressed as

$$
\operatorname{Pr}(D(\rho, T ; \pi) \geq \alpha) \leq \beta, \rho \sim\left\{X_{n}, n \geq 0\right\},
$$

where $\left\{X_{n}, n \geq 0\right\}$ is the $\pi$-induced Markov chain $M^{\pi}$.

We introduce the notion of mixing time and use it later to determine a stopping time $T$ for approximately satisfying time-unbounded specifications in PCTL.

Definition III.1 (Mixing time). Let $M$ be an MDP and let $\pi$ be an ergodic policy in $M$. Given $\epsilon \in \mathbb{R}_{+}$, the $\epsilon$-return mixing time of $\pi$ is the smallest $T$ such that for all $T^{\prime} \geq T$ , $\left|D\left(s, T^{\prime} ; \pi\right)-D(s, T ; \pi)\right| \leq \epsilon$.

Given a policy $\pi$, we can find an upper bound of this mixing time in terms of the second eigenvalue of the state transition $P^{\pi}\left(s^{\prime} \mid s\right)$ matrix of the Markov chain $M^{\pi}$ using methods in [18].

Next, we show how to translate PCTL formulas into chance constraints. We select the discount factor $\gamma=1$ unless otherwise specified. The reason is that PCTL considers the probability of satisfying path formulas and total cost without discounting. We distinguish three classes of PCTL.
1) Probabilistic formula $P_{\bowtie p}(\psi)$ : We consider a class of probabilistic formula $P_{\bowtie p}(\psi)$ where $\psi$ is a path formula of the form $\neg \phi_{1} \mathrm{U} \phi_{2}$, True $\boldsymbol{U} \phi$, or $\mathrm{X} \phi$, where $\phi, \phi_{1}$, and $\phi_{2}$ are propositional logic formulas. Formally,

$$
\left.s \models_{\pi} P_{\bowtie p}(\psi) \text { iff } P\left(\left\{\rho \in \operatorname{Path}^{\pi}(s)\right) \mid \rho \models \psi\right\}\right) \bowtie p .
$$

We show that a formula in this class can be represented by a chance constraint with a properly defined cost function.

Lemma III.1. Given a formula $P_{\bowtie p}(\mathrm{X} \phi)$, let's define a cost function $d: S \times A \rightarrow \mathbb{R}$ as $d(s, a)=\mathbf{E}_{s^{\prime} \sim P(\cdot \mid s, a)} \mathbf{1}\left(s^{\prime} \models \phi\right)$. Given a policy $\pi$, the $\pi$-induced Markov chain satisfies the formula, denoted $M^{\pi} \models P_{\bowtie p}(\mathrm{X} \phi)$, if and only if $D\left(X_{0}, T ; \pi\right) \bowtie p$ and the stopping time $T=2$.

Lemma III.2. Given a formula $P_{\bowtie p}(\operatorname{TrueU} \phi)$, let's define a cost function $d: S \times A \rightarrow \mathbb{R}$ as $d(s, a)=\mathbf{E}_{s^{\prime} \sim P(\cdot \mid s, a)} \mathbf{1}\left(s^{\prime} \mid=\right.$ $\phi)$ only if $s \not \models \phi$. Let all states in the set $\{s \mid s \models \phi\}$ be sink states. Given a policy $\pi$, the $\pi$-induced Markov chain satisfies the formula, denoted $M^{\pi} \mid=P_{\bowtie p}(\operatorname{TrueU} \phi)$, if it is one of the following cases:

- $₫ \in\{\geq,>\}: D\left(X_{0}, T_{\epsilon} ; \pi\right) \bowtie p$.

- $₫ \in\{\leq,<\}: D\left(X_{0}, T_{\epsilon} ; \pi\right) \bowtie p-\epsilon$.

where $\epsilon \in(0,1)$ is a small constant and $T_{\epsilon}$ is an upper bound of the $\epsilon$-mixing time of $\pi$.

Proof. By definition of cost function $d, D\left(X_{0} ; \pi\right)$ is the probability of eventually reaching a state satisfying $\phi$; $D\left(X_{0}, T ; \pi\right)$ is the probability of reaching a state satisfying $\phi$ within $T$ steps. Given $T_{\epsilon}$ is the mixing time, if a path of length $T_{\epsilon}$ has not yet visited a state that satisfies $\phi$, then the probability of satisfying the path formula as we continue along this path is less than $\epsilon$. Since the cost is nonnegative, we have $D\left(X_{0} ; \pi\right)-D\left(X_{0}, T_{\epsilon} ; \pi\right) \leq \epsilon$ for a predefined positive real number $\epsilon$. Next, we consider two cases: Case I: $\bowtie \in\{\geq,>\}, P_{\geq p}(\operatorname{TrueU} \phi)$ is equivalent to $D\left(X_{0} ; \pi\right) \geq p$. Given $D\left(X_{0} ; \pi\right) \geq D\left(X_{0}, T_{\epsilon} ; \pi\right)$, a sufficient condition for $D\left(X_{0} ; \pi\right) \geq p$ is that $D\left(X_{0}, T_{\epsilon} ; \pi\right) \geq p$. The same argument applies for strictly greater than, i.e., >. Case II: $\bowtie \in\{\leq,<\}, P_{<p}(\operatorname{TrueU} \phi)$ is equivalent to $D\left(X_{0} ; \pi\right) \leq p$. Given $D\left(X_{0} ; \pi\right)-D\left(X_{0}, T_{\epsilon} ; \pi\right) \leq \epsilon$, we have $D\left(X_{0} ; \pi\right) \leq D\left(X_{0}, T_{\epsilon} ; \pi\right)+\epsilon$. A sufficient condition for $D\left(X_{0} ; \pi\right) \leq p$ is that $D\left(X_{0}, T_{\epsilon} ; \pi\right)+\epsilon \leq p$, which is equivalent to $D\left(X_{0}, T_{\epsilon} ; \pi\right) \leq p-\epsilon$. The same argument applies for strictly less than, i.e., $<$.

Lemma III.3. Given a formula $P_{\bowtie p}\left(\neg \phi_{1} \mathrm{U} \phi_{2}\right)$, let's define a cost function $d: S \times A \rightarrow \mathbb{R}$ as $d(s, a)=\mathbf{E}_{s^{\prime} \sim P(\cdot \mid s, a)} \mathbf{1}\left(s^{\prime}=\right.$ $\left.\phi_{2}\right)$ only if $s \not \models \phi_{2}$. Let all states in the set $\left\{s|s|=\phi_{1} \vee \phi_{2}\right\}$ be sink states. Given a policy $\pi$, the $\pi$-induced Markov chain satisfies the formula, denoted $M^{\pi} \models P_{\bowtie p}\left(\neg \phi_{1} \cup \phi_{2}\right)$, if it is one of the following cases:

- $\bowtie \in\{\geq,>\}: D\left(X_{0}, T_{\epsilon} ; \pi\right) \bowtie p$.

- $\bowtie \in\{\leq,<\}: D\left(X_{0}, T_{\epsilon} ; \pi\right) \bowtie p-\epsilon$.

where $T_{\epsilon}$ is an upper bound of the $\epsilon$-mixing time of $\pi$.

Proof. The proof is similar to that of Lemma III.2. It is noted that when a system reaches a state satisfying $\phi_{1}$ prior 
to reaching a state satisfying $\phi_{2}$, it receives a cost of 0 even if the path continues to evolve until the time bound $T_{\epsilon}$.

2) Risk neutral cost constraint $C_{\bowtie m} \diamond^{\leq k} \phi: C_{\bowtie m} \diamond^{\leq k} \phi$ is the expected cost of paths satisfying $\nabla^{\leq k} \phi$. Based on the definition in [12], a policy $\pi$ satisfying the constraint given an initial state $s$, denoted $s \models_{\pi} C_{\bowtie m} \diamond^{\leq k} \phi$, if

$$
\underset{\pi}{\mathbf{E}}\left(s, X_{\diamond \leq k \phi}\right) \bowtie m,
$$

where $\underset{\pi}{\mathbf{E}}\left(s, X_{\diamond \leq k}\right)$ denotes the expectation of the random variable $X_{\diamond \leq k \phi}: \operatorname{Path}^{\pi}(s) \rightarrow \mathbb{R}_{\geq 0}$ with respect to the Markov chain $M^{\pi}$. Let $d: S \times \bar{A} \rightarrow \mathbb{R}$ be defined by $d(s, a)=\mathbf{E}_{s^{\prime} \sim P(\cdot \mid s, a)} \mathbf{1}\left(s^{\prime} \models \phi\right)$. For any state-action sequences $\rho=s_{0} a_{0} s_{1} a_{1} \ldots \in \operatorname{Path}^{\pi}(s), X_{\diamond \leq k}(\rho)=$ $D(\rho ; \pi)=\left(\sum_{t=0}^{T} d\left(s_{t}, a_{t}\right)\right)+\bar{D} \mathbf{1}\left(s_{T} \not \models \phi\right)$, where $T=$ $\min \left(k, \min \left\{j \mid s_{j} \models \phi\right\}\right)$ and $\bar{D} \gg 0$ is a penalty term, which is added when the path does not satisfy the specification ${ }^{\leq k} \phi$. Note that instead of assigning a cost of $\infty$ to a path that fails to satisfy $C_{\bowtie m} \diamond \leq k \phi$ in [12], we use a large penalty to ensure the planning problem is well-defined.

3) Risk sensitive PCTL: We consider a class of PCTL of the following from: $\phi_{1} \Longrightarrow \operatorname{Pr}_{\bowtie_{1} p}\left(X C_{\bowtie_{2} m} \diamond \leq k \phi_{2}\right)$ where $\phi_{1}$ and $\phi_{2}$ are propositional logic formulas, i.e., state formulas that use only conjunction and negation with atomic propositions in $\mathcal{A P}$. The risk-sensitive semantics of the formula means that if a Markov chain satisfies the formula, then starting from a state that satisfies $\phi_{1}$, in the next step the probability of a sampled path that satisfies $\nabla^{\leq k} \phi_{2}$ with a cost $\bowtie_{2} m$ is $\bowtie_{1} p$. Formally, it is defined by

$$
\begin{aligned}
s==_{\pi}\left(\phi_{1}\right. & \left.\Longrightarrow \operatorname{Pr}_{\bowtie_{1} p}\left(\mathrm{X} C_{\bowtie_{2} m} \nabla^{\leq k} \phi_{2}\right)\right) \text { iff } s \not \models \phi_{1} \\
& \text { or } s \models \phi_{1} \text { and } \operatorname{Pr}\left(\mathrm{X} C_{\bowtie_{2} m} \vee^{\leq k} \phi_{2}\right) \bowtie_{1} p .
\end{aligned}
$$

Lemma III.4. Given $\phi:=\phi_{1} \Longrightarrow \operatorname{Pr}_{\bowtie_{1} p}\left(X C_{\bowtie_{2} m} \diamond \leq k \phi_{2}\right)$, let's define a cost function $d: S \times A \rightarrow \mathbb{R}$ as $d(s, a)=$ $\mathbf{E}_{s^{\prime} \sim P(\cdot \mid s, a)} \mathbf{1}\left(s^{\prime}=\phi_{2}\right)$. Given a policy $\pi$, the $\pi$-induced Markov chain satisfies the formula, denoted $M^{\pi}=\phi$, if

$$
P\left(D(s, k+1 ; \pi) \bowtie_{2} m\right) \bowtie_{1} p, \quad \forall s=\phi_{1},
$$

where $D(s, k+1 ; \pi)=\left(\sum_{t=1}^{T} d\left(s_{t}, a_{t}\right)\right)+\bar{D} \mathbf{1}\left(s_{T} \not \models \phi_{2}\right)$, $T=\min \left(k+1, \min \left\{j \mid s_{j} \models \phi_{2}\right\}\right)$, and $D \gg 0$ is a penalty.

The proof is by construction and omitted. Further, let $Y=$ $\left\{s|s|=\phi_{1}\right\}$, the constraint is

$$
P\left(D(s, k+1 ; \pi) \bowtie_{2} m\right) \bowtie_{1} p, \text { for all } s \in Y .
$$

So far, we have shown that any PCTL formula in the three subclasses can be represented as a chance constraint with an appropriate definition of the cost function $d: S \times A \rightarrow \mathbb{R}$ of the following form: for some $\alpha \in \mathbb{R}$ and $\beta \in[0,1], k$

$$
\operatorname{Pr}(D(s, k ; \pi) \geq \alpha) \leq \beta, \quad \forall s \in Y,
$$

where $k$ is a positive integer and $Y \subseteq S$.

Remark 1. It is noted $D(s, k ; \pi)<\alpha$ is a special case of chance constraint and can be expressed by $\operatorname{Pr}(D(s, k ; \pi) \geq$ $\alpha) \leq 0$. The conjunction of multiple PCTLs can be translated into multiple chance constraints.
B. Formulating stochastic programming for PCTL constrained optimal planning

To develop an ADP method, we first replace the hardmax operator with the softmax operator [19], defined by

$$
\begin{gathered}
\mathcal{B} V(s)= \\
\tau \log \sum_{a} \exp \left\{\left(r(s, a)+\gamma \sum_{s^{\prime}} P\left(s^{\prime} \mid s, a\right) V\left(s^{\prime}\right)\right) / \tau\right\},
\end{gathered}
$$

where $\tau>0$ is a predefined temperature parameter. With the $\tau$ approaches 0, Eq. (2) recovers the hardmax Bellman operator. The softmax Bellman operator is contracting [19]. Given $V(\cdot ; \theta)$ the value function parameterized by $\theta$, one can obtain the corresponding state action function $Q(\cdot ; \theta)$ and policy $\pi(\cdot ; \theta)$ as:

$$
\begin{aligned}
& Q(s, a ; \theta)=r(s, a)+\gamma \sum_{s^{\prime}} P\left(s^{\prime} \mid s, a\right) V\left(s^{\prime} ; \theta\right), \\
& \pi(a \mid s ; \theta)=\exp ((Q(s, a ; \theta)-V(s ; \theta)) / \tau) .
\end{aligned}
$$

We denote $V^{*}$ the fixed point of the softmax operator, i.e., $\mathcal{B} V^{*}=V^{*}$.

Due to the monotonic contraction property of the softmax Bellman operator $\mathcal{B}$, it can be shown that for any value function $V$ that satisfies $\mathcal{B} V \leq V, V$ is an upper bound of the value function $V^{*}$. Given a value function approximation $V(s ; \theta)=\Phi(s) \theta$, the goal is to search for a function parameter $\theta \in \mathbb{R}^{\mathcal{K}}$ that solves the following optimization problem:

$$
\min _{\theta} \sum_{s \in S} c(s) V(s ; \theta)
$$

subject to: $\mathcal{B} V(s ; \theta)-V(s ; \theta) \leq 0, \quad \forall s \in S$,

$$
\operatorname{Pr}(D(s, k ; \theta) \geq \alpha)-\beta \leq 0, \quad \forall s \in Y .
$$

Next, we devise a trajectory-based value iteration algorithm for solving chance-constrained MDPs. Our approach is based on randomized optimization [20] that iteratively searches for, in the value function parameter space, an optimal parameter that minimizes a weighted distance between the upper bound given by the value function approximation and the true softmax value function.

We first introduce a continuous function $B: \mathbb{R} \rightarrow \mathbb{R}_{+}$ with support equal to $(0, \infty)$, in the sense that

$$
\begin{aligned}
& B(x)=0 \text { for all } x \in(-\infty, 0], \text { and } \\
& B(x)>0 \text { for all } x \in(0, \infty) .
\end{aligned}
$$

One such function is $B(x)=\max \{x, 0\}$. Let

$$
g(s ; \theta)=\mathcal{B} V(s ; \theta)-V(s ; \theta), \forall s \in S .
$$

Then, the constraints in 4a become $g(s ; \theta) \leq 0$ for all $s \in S$. Using randomized optimization [21], an equivalent representation of the set of constraints in $4 \mathrm{a}$ is

$$
\underset{\mathbf{s} \sim \Delta_{1}}{\mathbf{E}} B(g(\mathbf{s} ; \theta))=0
$$


where $\mathbf{s}$ is a random variable with a distribution $\Delta_{1}$ whose support is $S$. Similarly, let

$$
\ell(s ; \theta)=\operatorname{Pr}(D(s, K ; \theta) \geq \alpha)-\beta, \forall s \in Y .
$$

The equivalent representation of $4 \mathrm{~b}$ ) is

$$
\underset{\mathbf{s} \sim \Delta_{2}}{\mathbf{E}} B(\ell(\mathbf{s} ; \theta))=0,
$$

where $\mathbf{s}$ is a random variable with a distribution $\Delta_{2}$ over $Y$.

Thus, (4) is equivalent to:

$$
\min _{\theta} \sum_{s \in S} c(s) V(s ; \theta)
$$

subject to: $\underset{\mathbf{s} \sim \Delta_{1}}{\mathbf{E}} B(g(\mathbf{s} ; \theta))=0, \underset{\mathbf{s} \sim \Delta_{2}}{\mathbf{E}} B(\ell(\mathbf{s} ; \theta))=0$.

The choice of weights: : We choose the state relevant weight $c(s)=c(s ; \theta)$ to be the frequency with which different states are expected to be visited in the chain under policy $\pi(\cdot ; \theta)$, which is computed from $V(\cdot ; \theta)$ using (3). To justify the choice of this state relevant weight, it is noted that in the absence of chance constraints, we have the following optimization problem:

$$
\min _{\theta} \sum_{s \in S} c(s ; \theta) V(s ; \theta), \text { subject to: } \underset{\mathbf{s} \sim \Delta_{1}}{\mathbf{E}} B(g(\mathbf{s} ; \theta))=0 .
$$

The following result has been proved in [14] and rephrased with softmax Bellman operator.

Lemma III.5. [14] A vector $\theta^{*}$ solves

$$
\min _{\theta} \sum_{s \in S} c(s) \Phi \theta \text { subject to: } \mathcal{B} \Phi \theta-\Phi \theta \leq 0,
$$

if and only if it solves

$$
\min _{\theta} \sum_{s \in S}\left\|V^{*}-\Phi \theta\right\|_{1, c}, \text { subject to: } \mathcal{B} \Phi \theta-\Phi \theta \leq 0,
$$

where $\left\|V^{*}-\Phi \theta\right\|_{1, c}=\mathbf{E}_{\mathbf{s} \sim c}\left|\Phi(\mathbf{s}) \theta-V^{*}(\mathbf{s} ; \theta)\right|$.

For any state relevant weight $c \in \Delta(S)$, it holds that $\min _{\theta} \sum_{s \in S}\left\|V^{*}-\Phi \theta\right\|_{1, c} \geq \min _{\theta} \sum_{s \in S}\left\|V^{*}-\Phi \theta\right\|_{1, c(\cdot, \theta)}$ where $c(\cdot, \theta)$ is defined earlier. According to Theorem 1 of [14], the ideal weight is to choose $c$ that captures the (discounted) frequency with which different states are expected to be visited. For a given parameter $\theta$, the weight function $c(\cdot, \theta)$ can be obtained from on-policy sampling of trajectories with $\pi(\cdot ; \theta)$.

After formulating the optimization problem into stochastic programming problem, the augmented Lagrangian function of (5) is

$$
\begin{aligned}
& L_{\nu}(\theta, \lambda, \xi)=\sum_{s \in S} c(s ; \theta) V(s ; \theta)+\lambda \cdot \underset{\mathbf{s} \sim \Delta_{1}}{\mathbf{E}} B(g(\mathbf{s} ; \theta)) \\
& +\frac{\nu}{2} \cdot\left|\underset{\mathbf{s} \sim \Delta_{1}}{\mathbf{E}} B(g(\mathbf{s} ; \theta))\right|^{2}+\xi \cdot \underset{\mathbf{s} \sim \Delta_{2}}{\mathbf{E}} B(\ell(\mathbf{s} ; \theta)) \\
& +\frac{\nu}{2} \cdot\left|\underset{\mathbf{s} \sim \Delta_{2}}{\mathbf{E}} B(\ell(\mathbf{s} ; \theta))\right|^{2},
\end{aligned}
$$

where $\lambda$ and $\xi$ are the Lagrange multipliers and $\nu$ is a large penalty constant. Following the Quadratic Penalty function method [22], an optimal solution of (5) consists of solving a sequence of inner optimization problems of the form:

$$
\min _{\theta \in \mathbb{R}^{\mathcal{K}}} L_{\nu^{k}}\left(\theta, \lambda^{k}, \xi^{k}\right)
$$

where $\left\{\lambda^{k}\right\}$ and $\left\{\xi^{k}\right\}$ are sequences in $\mathbb{R},\left\{\nu^{k}\right\}$ is a positive penalty parameter sequence, and $\mathcal{K}$ is the size of $\theta$.

\section{Trajectory Sampling-based Approximate Value Iteration}

In this subsection, we devise a sampling-based method for solving (5). It is achieved by showing that the gradient of $L_{\nu^{k}}\left(\theta^{k}, \lambda^{k}, \xi^{k}\right)$ can be computed from sampled trajectories.

With a slight abuse of notation, let $M^{\theta}$ be a Markov chain induced by policy $\pi(\cdot ; \theta)$. By selecting $c(s ; \theta)=$ $\sum_{t=0}^{\infty} \operatorname{Pr}\left(X_{t}=s\right)$ the state visitation frequency in the Markov chain $M^{\theta}$, for an arbitrary function $f: S \rightarrow \mathbb{R}$, it holds that

$$
\sum_{s \in S} c(s ; \theta) f(s ; \theta)=\int p(h ; \theta) f(h ; \theta) d h,
$$

where $p(h ; \theta)$ is the probability of path $h$ in the Markov chain $M^{\theta}, f(h ; \theta)=\sum_{i=1}^{|h|} f\left(s_{i} ; \theta\right)$.

Furthermore, by selecting $\Delta_{1} \propto c(\cdot ; \theta)$ and letting $f(s ; \theta)=V(s ; \theta)+\lambda^{k} \cdot B(g(s ; \theta))+\frac{\nu^{k}}{2} \cdot|B(g(s ; \theta))|^{2}$, and $m\left(\Delta_{2} ; \theta\right)=\xi^{k} \cdot \underset{\mathbf{s} \sim \Delta_{2}}{\mathbf{E}} B(\ell(\mathbf{s} ; \theta))+\frac{\nu^{k}}{2} \cdot\left|\underset{\mathbf{s} \sim \Delta_{2}}{\mathbf{E}} B(\ell(\mathbf{s} ; \theta))\right|^{2}$, the $k$-th objective function in $(8)$ becomes

$$
\min _{\theta} \underbrace{\int p(h ; \theta) f(h) d h}_{F(\theta)}+m\left(\Delta_{2} ; \theta\right) .
$$

Using the gradient descent, parameter $\theta$ is updated by

$$
\theta^{j+1} \leftarrow \theta^{j}-\eta_{1} \cdot \nabla_{\theta} F(\theta)-\eta_{2} \cdot \nabla_{\theta} m\left(\Delta_{2} ; \theta\right),
$$

where $j$ represent the $j$-th inner iteration, $\eta_{1}$ and $\eta_{2}$ are positive step sizes.

$$
\nabla_{\theta} F(\theta)=\int \underbrace{\nabla_{\theta} p(h ; \theta) f(h ; \theta) d h}_{1}+\int \underbrace{p(h ; \theta) \nabla_{\theta} f(h ; \theta) d h}_{2},
$$

where

$$
\begin{aligned}
1 & =\int \nabla_{\theta} p(h ; \theta) f(h ; \theta) d h \\
& =\int p(h ; \theta) \nabla_{\theta} \log p(h ; \theta) f(h ; \theta) d h \\
& =\int p(h ; \theta)\left[\sum_{t=0}^{|h|} \nabla_{\theta} \log \pi\left(a_{t} \mid s_{t} ; \theta\right)\right] f(h ; \theta) d h \\
& \approx \frac{1}{N_{h}} \sum_{h \sim p(h ; \theta)}\left[\sum_{t=0}^{|h|} \nabla_{\theta} \log \pi\left(a_{t} \mid s_{t} ; \theta\right)\right] f(h ; \theta) .
\end{aligned}
$$

(Monte-Carlo approximation)

Variance reduction [23] can be employed to reduce the error variance in the Monte-Carlo approximation. 


$$
\begin{aligned}
2 & =\int p(h ; \theta) \nabla_{\theta} f(h ; \theta) d h=\int p(h ; \theta)\left[\sum_{t=0}^{|h|} \nabla_{\theta} f\left(s_{t} ; \theta\right)\right] d h \\
& \approx \frac{1}{N_{h}} \sum_{h \sim p\left(s_{t} ; \theta\right)}\left[\sum_{t=0}^{|h|} \nabla_{\theta} f\left(s_{t} ; \theta\right)\right],
\end{aligned}
$$

where $\nabla_{\theta} f\left(s_{t} ; \theta\right)=\nabla_{\theta} V\left(s_{t} ; \theta\right)+\lambda^{k}$. $\nabla_{g} B\left(g\left(s_{t} ; \theta\right)\right) \nabla_{\theta} g\left(s_{t} ; \theta\right)+\nu^{k} \cdot B\left(g\left(s_{t} ; \theta\right) \nabla_{\theta} g\left(s_{t} ; \theta\right)\right.$. Note if $B(x)=\max \{x, 0\}$, then $\nabla_{g} B(x)= \begin{cases}1 & \text { if } x>0, \\ 0 & \text { otherwise. }\end{cases}$

In term of the derivative of the second term:

$$
\begin{aligned}
\nabla_{\theta} m\left(\Delta_{2} ; \theta\right) & =\xi^{k} \cdot \underset{\mathbf{s} \sim \Delta_{2}}{\mathbf{E}} \nabla_{\ell} B(\ell(\mathbf{s} ; \theta)) \nabla_{\theta} \ell(\mathbf{s} ; \theta) \\
& +\nu^{k} \underset{\mathbf{s} \sim \Delta_{2}}{\mathbf{E}} \cdot B(\ell(\mathbf{s} ; \theta)) \nabla_{\ell} B(\ell(\mathbf{s} ; \theta)) \nabla_{\theta} \ell(\mathbf{s} ; \theta) .
\end{aligned}
$$

We generate a set $Z$ of trajectories starting at a state with the initial distribution $\Delta_{2}$ to estimate the gradient. A simple choice of $\Delta_{2}$ is a uniform distribution over $Y$. Let $Z(s)$ be a set of trajectories in $Z$ with the initial state $s$ and $D(z)$ be the total cost along the trajectory $z$, we have

$$
\begin{aligned}
\nabla_{\theta} \ell(s ; \theta) & \approx \nabla_{\theta}\left[\sum_{z \in Z(s)} p(z ; \theta) \mathbf{1}\{D(z) \geq \alpha\}-\beta\right] \\
& =\sum_{z \in Z(s)} \nabla_{\theta} p(z ; \theta) \mathbf{1}\{D(z) \geq \beta\} \\
& =\sum_{z \in Z(s)} p(z ; \theta \mid s) \nabla_{\theta} \log p(z ; \theta) \mathbf{1}\{D(z) \geq \alpha\} \\
& \approx \frac{1}{|Z(s)|} \sum_{z \in Z(s)} \nabla_{\theta} \log p(z ; \theta) \mathbf{1}\{D(z) \geq \alpha\}
\end{aligned}
$$

where $\nabla_{\theta} \log p(z ; \theta)=\sum_{t=0}^{|z|} \nabla_{\theta} \log \pi\left(a_{t} \mid s_{t} ; \theta\right)$.

$$
\begin{aligned}
B(\ell(s ; \theta)) & \approx B\left[\sum_{z \in Z(s)} p(z ; \theta) \mathbf{1}\{D(z) \geq \alpha\}-\beta\right] \\
& =\max \left(\sum_{z \in Z(s)} p(z ; \theta \mid s) \mathbf{1}\{D(z) \geq \alpha\}-\beta, 0\right) \\
& \approx \max \left(\frac{1}{|Z(s)|} \sum_{z \in Z(s)} \mathbf{1}\{D(z) \geq \alpha\}-\beta, 0\right) .
\end{aligned}
$$

Finally, the gradient $\nabla_{\theta} m\left(\Delta_{2} ; \theta\right)$ is approximated by Monte Carlo approximation. The sample bound $|Z|$ is determined by methods in stochastic programming [24]. Using the gradient descent algorithm we can update $\theta^{j}$ to $\theta^{j+1}$ until $\left\|\theta^{j+1}-\theta^{j}\right\| \leq \epsilon$. The step-sizes are updated using the square summable step rule [25], that is, $\eta_{i}^{k+1}=\frac{\eta_{i}^{k}}{k}, \quad$ for $i=1,2$.

After the inner optimization for 8 converges, we update formula for multipliers $\lambda$ and $\xi$ as

$$
\begin{aligned}
& \lambda^{k+1}=\lambda^{k}+\nu^{k} \cdot \underset{\mathbf{s} \sim \Delta_{1}}{\mathbf{E}} B\left(g\left(\mathbf{s} ; \theta^{k}\right)\right) ; \\
& \xi^{k+1}=\xi^{k}+\nu^{k} \cdot \underset{\mathbf{s} \sim \Delta_{2}}{\mathbf{E}} B\left(\ell\left(\mathbf{s} ; \theta^{k}\right)\right),
\end{aligned}
$$

where the expectations are approximated by Monte-Carlo methods. The penalty value $\nu$ is updated using the rule in [22].

$$
\nu^{k+1}= \begin{cases}b \nu^{k} & \| \mathbf{E}_{\mathbf{s} \sim \Delta_{2}} B\left(\ell ( \mathbf { s } ; \theta ^ { k } ) \| > \rho \| \mathbf { E } _ { \mathbf { s } \sim \Delta _ { 2 } } B \left(\ell\left(\mathbf{s} ; \theta^{k-1}\right) \|\right.\right. \\ & \text { or } \\ & \| \mathbf{E}_{\mathbf{s} \sim \Delta_{1}} B\left(g ( \mathbf { s } ; \theta ^ { k } ) \| > \rho \| \mathbf { E } _ { \mathbf { s } \sim \Delta _ { 1 } } B \left(g\left(\mathbf{s} ; \theta^{k-1}\right) \|\right.\right. \\ \nu^{k} & \text { otherwise, }\end{cases}
$$

where $b>1$ to ensure the sequence $\nu^{k}$ are non-increasing and a typical choice of $\rho$ is 0.25 . Note that a different penalty parameter $\nu_{i}^{k}$ can be chosen for each constraint. For example, $\nu_{1}^{k}$ is chosen for constraint (4a) and $\nu_{2}^{k}$ is chosen for constraint 4b]. Furthermore, the update of penalty parameter does not require any new samples, since $\mathbf{E}_{\mathbf{s} \sim \Delta_{1}} B\left(g\left(\mathbf{s} ; \theta^{k}\right)\right.$ and $\mathbf{E}_{\mathbf{s} \sim \Delta_{2}} B\left(\ell\left(\mathbf{s} ; \theta^{k}\right)\right.$ can be evaluated based on sampled trajectories.

The following assumptions are required in the analysis of the convergence.

A1 For $y \in\left\{\eta_{1}, \eta_{2}\right\}, y^{k}>0$ for each $k \geq 1, \sum_{k=1}^{\infty} y^{k}=$ $\infty$, and $\sum_{n=1}^{\infty}\left(y^{k}\right)^{2}<\infty$.

A2 The value function approximation is continuously differentiable in $\theta$ and $\nabla_{\theta} V(\cdot ; \theta)$ is locally Lipschitz continuous.

A3 There exists a feasible solution for (5).

A4 For time-unbounded PCTL, the length of a sampled trajectory is lower bounded by either the time bound in PCTL formulas or the mixing time of the Markov chain with policy parameterized by $\theta$.

Theorem III.6. Assuming A1-A4, the sequence of value function updates converges almost surely (with probability 1) to a local optimal solution $\theta^{*}$ for the chance-constrained optimization problem in (5).

The convergence proof is standard for stochastic programming and omitted for space limitation.

\section{CAse Studies}

We validate the algorithm in two motion planning problems modeled as stochastic gridworld problems, illustrated in Fig.11: A simple reach-avoid task (a), and a planning problem with a PCTL constraint (b). In each state $s \in S$ and for robot's different actions (heading up ('U'), down ('D'), left ('L'), right ('R')), the probability of arriving at the correct cell is $1-0.1 \times N$, where $N$ is the number of the neighbors of the current state including itself. If the system hits the wall, it will be bounced back to its original cell.

\section{A. Planning without PCTL constraints}

Without considering any constraints, the first experiment is designed to observe the the relation between the weighting parameters and the approximation error and to justify the choice of weights in (4). The planning objective is to find an approximately optimal policy which drives the robot from the initial position $s_{\text {init }}:[0,0]$ to the goal $s_{\text {goal }}:[8,10]$ while avoiding the obstacles. The reward is defined as the following: the robot receives a reward of 100 if $P\left(s_{\text {goal }} \mid\right.$ $s, a)>0.5$. 


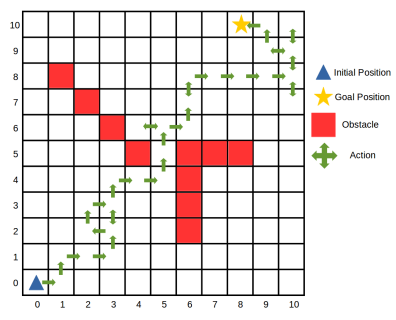

(a)

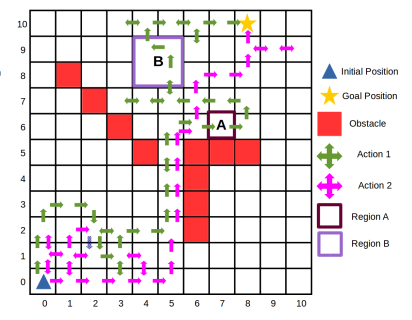

(b)
Fig. 1: (a) Gridworld with initial (triangle), goal (star), obstacles(solid squares), no PCTL constraints. (b) The gridworld with PCTL constraint $A \Longrightarrow \operatorname{Pr}_{\geq \delta}\left(C_{\leq 13} \oslash^{\leq 15} B\right)$ where $A$ and $B$ are regions marked in the graph.

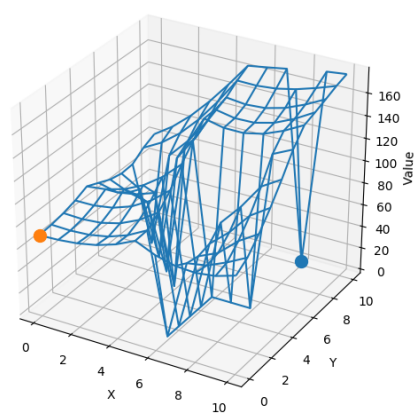

(a)

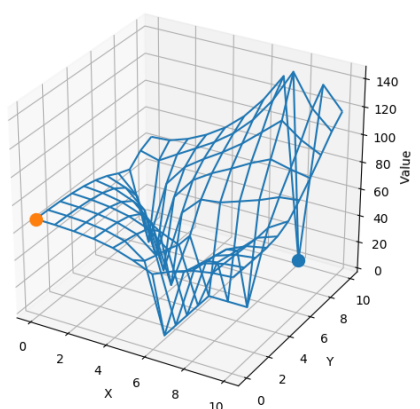

(b)
Fig. 2: Value Functions. (a) Approximate value function obtained with the proposed ADP method. (b) The true value function obtained with softmax value iteration.

The value function approximation is $V(s ; \theta)=\Phi \theta$, where the basis functions $\Phi=\left[\phi_{1}, \phi_{2}, \ldots, \phi_{\mathcal{K}}\right]^{\top}$ are Geodesic Gaussian Kernels (GGKs) [26] defined as the following: $\Phi_{j}(s)=K\left(s, c^{(j)}\right)$ and $K\left(s, s^{\prime}\right)=\exp \left(-\frac{S P\left(s, s^{\prime}\right)^{2}}{2 \sigma^{2}}\right)$, where $\left\{c^{(j)}, j=1, \ldots, \mathcal{K}\right\}$ is a set of preselected centers. In this example, we select the centers to be $\{(x, y) \mid x, y \in$ $\{0,5,10\}\}$ and the variance $\sigma$ to be 5 . The term $S P\left(s, s^{\prime}\right)$ refers to the shortest path from state $s$ to state $s^{\prime}$ in the graph, assuming deterministic transitions.

The parameters used in the algorithm are the following (with respect to Section III-C): the temperature parameter $\tau=5, b=1.1, \eta_{1}=0.1$, the initial penalty parameter $\nu^{0}=10.0$, the initial Lagrangian multipliers $\lambda^{0}=0$. During each iteration, 30 trajectories of length $\leq 6$ are sampled. The stopping criterion for each inner optimization problem is $\left\|\nabla_{\theta} L_{\nu^{k}}\left(\theta^{j}, \lambda^{k}\right)\right\| \leq \epsilon^{k}$, where $\left\{\epsilon^{k}\right\}$ is a positive sequence converging to 0 . In a single run, the algorithm converges after 4 outer iterations, with 164,24,8,1 iterations for each outer iteration, respectively. Fig. 3 shows the result of 100 independent experiments for solving the same planning problem starting with the same initial $\theta$ which is a zero vector. The black line represents the mean, the shaded area is limited by the maximum and minimum values over iterations, and the red line is the ground truth. The black line is always above the red line. This is expected as the value function approximation is an upper bound of the optimal value function.

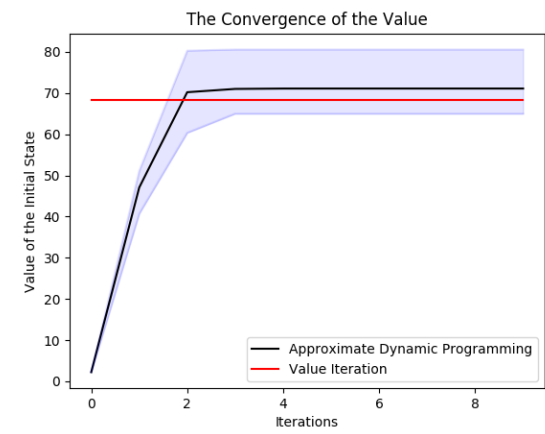

Fig. 3: The learning curve for the stochastic gridworld, averaged across 100 runs of the ADP algorithm with random initialization.

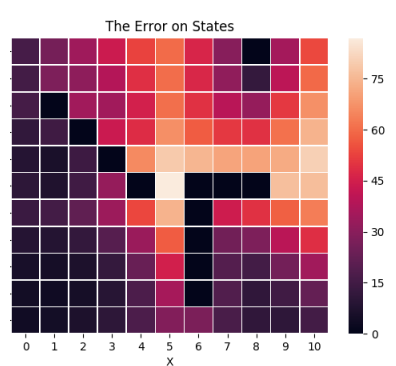

(a)

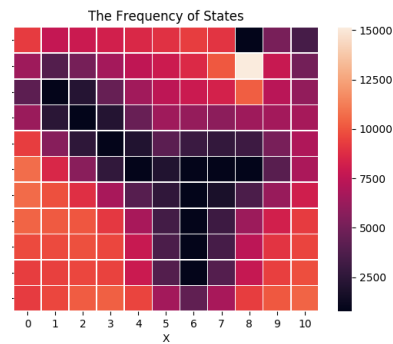

(b)
Fig. 4: Comparison of state visitation frequency and the approximation error for different states (a) The heatmap of the error $V\left(s ; \theta^{*}\right)-V^{*}(s)$. (b) The heatmap of state visitation frequencies under policy $\pi(\cdot ; \theta)$.

To illustrate the approximation error, we compare the optimal and approximate-optimal value functions in Fig. 4a, which plots the error $V(s ; \theta)-V(s)^{*}$ on each individual state $s$. Fig. $4 \mathrm{~b}$ shows state visitation frequency under the computed policy. It shows that for a state with a high visitation frequency under the optimal policy, the error tends to be very small. This result is expected due to our choice of weight parameters that have larger weights for states with high visitation frequencies. Fig. 1a shows one run generated by following the computed policy.

\section{B. Planning with PCTL constraints}

Consider including a PCTL constraint $A \Longrightarrow$ $\operatorname{Pr}_{\geq \delta}\left(\mathrm{X} C_{\leq 13} \diamond \leq 14 B\right)$ : When the agent visits $A$, then it will ensure, starting from the next state, with a probability at least 0.2 , to eventually visit region $B$ in less than 14 steps with a cost less than 13. Region A and B are shown in Fig. $1 \mathrm{~b}$ Let $d: S \times A \rightarrow \mathbb{R}$ be defined by $d(s, a)=1$.

We use the same value function approximation, the same stopping criterion for the inner optimization, and the same set of parameters with different initial penalty parameters $\nu_{1}^{0}=10.0$ and $\nu_{2}^{0}=500$ for constraints $4 \mathrm{a}$ and $4 \mathrm{~b}$, respectively. For each iteration, there are 30 trajectories (of lengths $\leq 6$ ) sampled and another 100 trajectories (of lengths $=15)$ starting from the region $\mathrm{A}$ for the chance constraints. Given $\delta=0.2$, Fig. 5 shows the convergence of the parameters. Even by adding PCTL constraints, the 


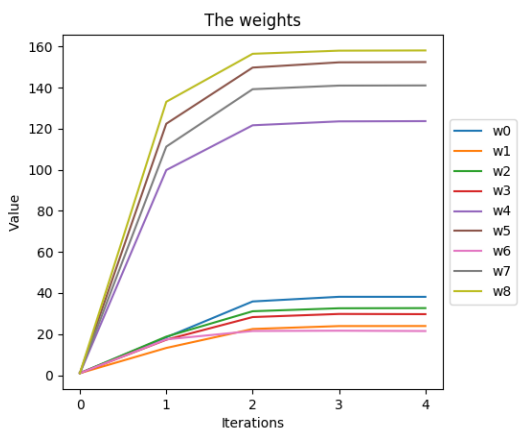

Fig. 5: The Convergence of Parameters

TABLE I: Frequencies of satisfying paths under different $\delta$.

\begin{tabular}{ccccc}
\hline$\delta$ & 0 & 0.1 & 0.2 & 0.3 \\
Num. of satisfying paths & 3130 & 2227 & 4581 & 7410 \\
\% of satisfying paths & 0.15 & 0.11 & 0.23 & 0.37 \\
\hline
\end{tabular}

algorithm converges after 4 outer iterations. Fig. $1 \mathrm{~b}$ shows two trajectories simulated by following the computed policy. In this one sampled trajectory, the system reaches $\mathrm{A}$ and then $\mathrm{B}$ with a cost less than the given threshold. In another sampled trajectory, the system does not visit region A and directly goes to the goal.

We tested the algorithm with different values for the $\delta$. Table. I shows the frequencies of trajectories satisfying the cost constraint $X C_{\leq 13}{ }^{\leq 14} B$ under different values for $\delta$ after the empirical evaluation of 20000 trajectories starting from $A$. In all experiments, the PCTL constraint is satisfied. The result shows that as $\delta$ increases, the probability of satisfying the constraint also increases, but not monotonically. This can be caused by the chosen function approximation.

\section{CONCLUSION}

We have presented an approximate value iteration method for MDP with PCTL constraints. We proposed a method that translates PCTL constraints into chance constraints and uses stochastic programming for solving an upper bound of the optimal value function subject to constraints in PCTL. The almost sure convergence of the proposed algorithm is guaranteed under several assumptions. There are several future directions enabled by this study: First, the current method only studies a class of PCTL for which memoryless policies are sufficient to be approximately optimal. We are interested in extending this method to a large class of temporal logic formulas, for which finite-memory is needed for optimality. Second, it is possible to develop distributed ADP using approximate value iteration based on decomposition-based planning in large-scale MDPs.

\section{REFERENCES}

[1] X. Ding, S. L. Smith, C. Belta, and D. Rus, "Optimal control of markov decision processes with linear temporal logic constraints," IEEE Transactions on Automatic Control, vol. 59, no. 5, pp. 1244 1257, 2014.

[2] J. Fu and U. Topcu, "Probably approximately correct mdp learning and control with temporal logic constraints," in Proceedings of Robotics: Science and Systems, (Berkeley, USA), July 2014.
[3] J. Wang, X. Ding, M. Lahijanian, I. C. Paschalidis, and C. A. Belta, "Temporal logic motion control using actor-critic methods," The International Journal of Robotics Research, vol. 34, no. 10, pp. 13291344, 2015.

[4] J. Fu and U. Topcu, "Computational methods for stochastic control with metric interval temporal logic specifications," in Decision and Control (CDC), 2015 IEEE 54th Annual Conference on, pp. 74407447, IEEE, 2015.

[5] M. Lahijanian, S. B. Andersson, and C. Belta, "Temporal logic motion planning and control with probabilistic satisfaction guarantees," IEEE Transactions on Robotics, vol. 28, no. 2, pp. 396-409, 2012.

[6] M. Lahijanian, S. Andersson, and C. Belta, "Control of markov decision processes from pctl specifications," in American Control Conference (ACC), 2011, pp. 311-316, IEEE, 2011.

[7] D. Sadigh, E. S. Kim, S. Coogan, S. S. Sastry, and S. A. Seshia, "A learning based approach to control synthesis of markov decision processes for linear temporal logic specifications," in Decision and Control (CDC), 2014 IEEE 53rd Annual Conference on, pp. 10911096, IEEE, 2014.

[8] R. Dimitrova, J. Fu, and U. Topcu, "Robust optimal policies for markov decision processes with safety-threshold constraints," in $D e$ cision and Control (CDC), 2016 IEEE 55th Conference on, pp. 70817086, IEEE, 2016.

[9] S. Junges, N. Jansen, C. Dehnert, U. Topcu, and J.-P. Katoen, "Safetyconstrained reinforcement learning for mdps," in International Conference on Tools and Algorithms for the Construction and Analysis of Systems, pp. 130-146, Springer, 2016.

[10] D. P. Bertsekas et al., Dynamic programming and optimal control, vol. 1. Athena Scientific Belmont, Massachusetts, 1996.

[11] D. P. Bertsekas, "Neuro-dynamic programming," in Encyclopedia of optimization, pp. 2555-2560, Springer, 2008.

[12] M. Kwiatkowska, G. Norman, and D. Parker, "Stochastic model checking," in International School on Formal Methods for the Design of Computer, Communication and Software Systems, pp. 220-270, Springer, 2007.

[13] Y. Chow, M. Ghavamzadeh, L. Janson, and M. Pavone, "Riskconstrained reinforcement learning with percentile risk criteria," Journal of Machine Learning Research, vol. 18, no. 167, pp. 1-51, 2018.

[14] D. P. De Farias and B. Van Roy, "The linear programming approach to approximate dynamic programming," Operations research, vol. 51, no. 6, pp. 850-865, 2003.

[15] P. Geibel, "Reinforcement learning for mdps with constraints," in European Conference on Machine Learning, pp. 646-653, Springer, 2006.

[16] M. L. Puterman, Markov decision processes: discrete stochastic dynamic programming. John Wiley \& Sons, 2014.

[17] R. Fagin, J. Y. Halpern, and N. Megiddo, "A logic for reasoning about probabilities," Information and computation, vol. 87, no. 1-2, pp. 78128, 1990.

[18] A. Sinclair, Algorithms for random generation and counting: a Markov chain approach. Springer Science \& Business Media, 2012.

[19] R. S. Sutton, A. G. Barto, et al., Reinforcement learning: An introduction. MIT press, 1998.

[20] R. Tempo, G. Calafiore, and F. Dabbene, Randomized algorithms for analysis and control of uncertain systems: with applications. Springer Science \& Business Media, 2012.

[21] V. B. Tadić, S. P. Meyn, and R. Tempo, "Randomized algorithms for semi-infinite programming problems," in Probabilistic and Randomized Methods for Design under Uncertainty, pp. 243-261, Springer, 2006.

[22] D. P. Bertsekas, Nonlinear programming. Athena scientific Belmont, 1999.

[23] R. J. Williams, "Simple statistical gradient-following algorithms for connectionist reinforcement learning," Machine learning, vol. 8, no. 34, pp. 229-256, 1992.

[24] D. P. De Farias and B. Van Roy, "On constraint sampling in the linear programming approach to approximate dynamic programming," Mathematics of operations research, vol. 29, no. 3, pp. 462-478, 2004.

[25] S. Boyd, L. Xiao, and A. Mutapcic, "Subgradient methods," lecture notes of EE392o, Stanford University, Autumn Quarter, vol. 2004, pp. 2004-2005, 2003.

[26] M. Sugiyama, Statistical reinforcement learning: modern machine learning approaches. Chapman and Hall/CRC, 2015. 